\title{
Riesgos psicosociales y actitudes sobre prácticas sexuales bajo el efecto del alcohol o drogas en adolescentes de la ciudad de Medellín*
}

\author{
Psychosocial Risk and Attitudes about Sexual Practices with \\ Alcohol or Drugs Effects in Adolescents at Medellín
}

Recibido: enero 20 de 2012 | Recibido: octubre 28 de 2012 | Aceptado: diciembre 20 de 2012

\author{
Olber EdUARdo Arango-Tobón ** \\ Guillermo Alonso Castaño Pérez \\ SANDRA QUINTERO \\ CAROLINA MONTOYA MONTOYA \\ SANTIAGO MORALES MESA *** \\ AlEXANDER RODRÍGUEZ BUSTAMANTE **** \\ Fundación Universitaria Luis Amigó, Medellín, Colombia
}

doi:10.11144/Javeriana.UPSY12-3.rpap

Para citar este artículo: Arango, O. E., Castaño, G. A., Quintero, S., Montoya, C., Morales, S. \& Rodríguez, A. (2013). Riesgos psicosociales y actitudes sobre prácticas sexuales bajo el efecto del alcohol o drogas en adolescentes de la ciudad de Medellín. Universitas Psychologica, 12(3), 887-898. doi:10.11144/Javeriana.UPSY12-3.rpap

* Artículo original producto de la investigación denominada "Consumo de drogas y prácticas sexuales en adolescentes entre 14 y 17 años de la ciudad de Medellín en el año 2011", subvencionada por la Fundación Universitaria Luis Amigó.

** Fundación Universitaria Luis Amigó, Medellín, Colombia. Facultad de Psicología y Ciencias Sociales. E-mails: eduarango@une.net.co,olber.arangoto@ amigo.edu.co,gcastano@funlam.edu.co, Sandra. quinterolo@une.net.co, cmontoya@funlam.edu. co. Researchers ID: H-6661-2013, H-6673-2013, H-6670-2013, H-6689-2013.

**** Fundación Universitaria Luis Amigó, Medellín, Colombia. Facultad de Derecho y Ciencias Políticas. E-mail:smorales@funlam.edu.co

****** Fundación Universitaria Luis Amigó, Medellín, Colombia. Programa de Desarrollo Familiar. Email: alexander.rodriguezbu@amigo.edu.co

\section{RESUMEN}

En este artículo se analizan las actitudes cognitivas y los factores psicosociales relacionados con las prácticas sexuales bajo el efecto de alcohol y drogas en adolescentes de la ciudad de Medellín. El tipo de estudio fue no experimental, de nivel descriptivo y correlacional. La muestra estuvo conformada por 955 estudiantes de grados 9. $\stackrel{\circ}{ }, 10 .^{\circ}$ y $11 .^{\circ}$ de colegios públicos y privados de la ciudad de Medellín. La edad, el sexo, el tipo de familia, las personas encargadas de la crianza, las normas al interior de la familia, la influencia del grupo de pares y las actitudes cognitivas y afectivas juegan un rol importante en la realización de prácticas sexuales riesgosas bajo el consumo del alcohol y las drogas durante la adolescencia.

Palabras clave autores

Factores psicosociales, prácticas sexuales, consumo de alcohol y drogas, actitudes cognitivas, adolescencia.

Palabras clave descriptores

Psicología de la salud, sustancias psicoactivas, investigación cuantitativa.

\section{A B S T R A C T}

In this article we examined some psychosocial factors and cognitive attitudes related to sexual practices under the influence of alcohol and drugs among adolescents in the city of Medellín in 2011. The type of study was non-experimental with a descriptive and correlational level. The sample consisted of 955 students in grades 9,10 and 11 public and private colleges. Age, sex, type of family, persons responsible for raising, group influence and cognitive and affective attitudes; play an important role in the performance of risky sexual practices on the use of alcohol and drugs during adolescence. Key words authors

Psychosocial Factors, Sexual Practices, Alcohol and Drugs Use, Cognitive Attitudes, Teens.

Key words plus

Health Psychology, Psychoactive Drugs, Quantitative Research. 


\section{Introducción}

Algunas características personales como el sistema de creencias, reflejados en las actitudes cognitivas $\mathrm{y}$ afectivas, parecen ser un factor de riesgo importante a la hora de tomar decisiones sobre los beneficios y ventajas de usar drogas y realizar prácticas sexuales bajo sus efectos (Ajzen \& Fishbein, 2008). Desde nuestro marco referencial, entendemos las actitudes como un proceso cognitivo que implica la evaluación y valoración con respecto a un objeto o situación (Ajzen \& Cote, 2008).

Algunos estudios han mostrado que las creencias y actitudes de los adolescentes son un factor determinante a la hora de tomar decisiones que los llevan a realizar conductas de riesgo (Abasiubong, Udoh, Ntuk, Eknem \& Etukumana, 2011; Caldeira et al., 2009; Goncalvez, Silveira \& Bartex, 2009; Orchowski \& Barnett, 2012). En esta línea, Ceballos y Campo-Arias (2005) realizaron un estudio en Santa Marta (Colombia) con una muestra de 223 adolescentes entre los 13 y 17 años, estudiantes de colegios privados y públicos. Estos autores encontraron que el uso del condón está directamente relacionado con el género, edad y las creencias por género en relación con el uso de los anticonceptivos.

En otro estudio realizado por Sierra, Pérez, Pérez y Núñez (2005), con 1.492 estudiantes con edades entre 13 y 22 años y de estratos bajos de la ciudad de Bogotá, los autores sugieren que el consumo de sustancias psicoactivas (SPA) está directamente relacionado con la creencia de los adolescentes respecto a las mismas. Los consumidores de SPA perciben estas sustancias como poco nocivas, creen que, además de sensaciones placenteras, les procuran una evasión de la realidad. Mientras que los no consumidores de SPA manifestaron actitudes más cautelosas y preocupación por las consecuencias. Algunos autores consideran que los preconceptos y la valoración de las SPA, así como la baja percepción de riesgo frente a ellas, sus efectos y consecuencias, los convierte en un importante factor de riesgo para su uso y abuso (Echeverría, 2009; Kafaar, Swartz, Kagee, Lesch \& Jaspan, 2007; Meneses et al., 2009; Varela, Salazar, Cáceres \& Tovar, 2007).
Algunos estudios han reportado la incidencia de factores psicosociales de riesgo, relacionando, entre otras conductas, el consumo de sustancias psicoactivas durante la adolescencia (Campo, 2009; Campo, Ceballo \& Henazo, 2010; Jessor, 1991; Jessor \& Jessor, 1977; L'Engle \& Jackson, 2008; Patrick \& Maggs, 2009). En esos estudios se hace alusión a características cognitivas del individuo, variables sociales, familiares y del contexto que describen, explican y predicen la aparición de conductas relacionadas con el uso y el abuso de drogas durante la adolescencia (Bertoni et al., 2009; Calsyn et al., 2010; Kafaar et al., 2007; L'Engle \& Jackson, 2008; Lameiras, Failde, Bimbela \& Alfaro, 2008; Mann, Harmoni \& Power, 1989).

En síntesis, según los estudios citados anteriormente, se pueden establecer relaciones entre la dimensión cognitiva (creencias y actitudes) y las conductas relacionadas con el consumo de SPA y prácticas sexuales bajo sus efectos. También se pudo apreciar la incidencia de otras variables como la familia y la relación con los otros (pares y amigos) en la decisión de consumir drogas o de tener prácticas sexuales y cómo desde el entorno familiar y la interacción con los otros se pueden formar creencias y actitudes que dirigirán la toma de decisiones, las conductas y las emociones vinculadas con las prácticas sexuales bajo el efecto de SPA.

La importancia y novedad de este estudio radica en el conocimiento y profundización del rol que juegan algunos factores personales; especialmente factores cognitivos y afectivos, factores familiares y del entorno, en la probabilidad de desarrollar conductas de riesgo relacionadas con las prácticas sexuales bajo el consumo de SPA durante la adolescencia. En este sentido, la mayoría de investigaciones encontradas sobre el tema, han girado en torno a prevalencias del consumo de SPA o prácticas sexuales, observando el fenómeno de manera aislada. El presente estudio, por el contrario, intenta establecer una relación entre el consumo de SPA y cómo puede esto llevar a prácticas sexuales de riesgo en los adolescentes. Así mismo, y de acuerdo con los hallazgos, se plantea la posibilidad de proponer modelos de prevención en el consumo SPA y de promoción de prácticas sexuales saludables de los adolescentes desde un enfoque cognitivo. El 
objetivo de esta investigación fue identificar las actitudes cognitivas/afectivas y factores psicosociales que predisponen a los adolescentes a realizar prácticas sexuales bajo los efectos de SPA. La hipótesis central de la investigación sugiere que las actitudes cognitivas y afectivas son un factor de riesgo en la aparición de prácticas sexuales azarosas bajo el efecto de SPA.

\section{Método}

\section{Participantes}

Se determinó la muestra mediante método probabilístico aleatorio y por conglomerados. Se tomó como universo poblacional el número total de estudiantes

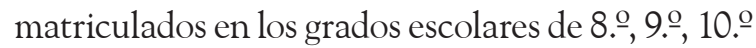
y 11. en el año 2010 de colegios públicos y privados de la ciudad de Medellín, con edades comprendidas entre los 14 y 17 años; el total del universo poblacional fue de 91.857 estudiantes. El tamaño muestral incluido fue de 955 estudiantes, usando un intervalo de confianza del $95 \%$. En la Tabla 1 se describen las variables demográficas de la muestra: el $54.8 \%$ son hombres y el $45.2 \%$ mujeres, con edades comprendidas entre los 14 y los 18 años, la mayoría entre los 15 y los 16 años; hay un mayor número de estratos socioeconómicos bajos 1, 2 y 3 (69.43\%), de colegios públicos $(68.9 \%$ ) y matriculados en los grados 9.․ (33.3 \%), 10. (29.3 \%) y 11.․ (30.4\%).

\section{Diseño}

El tipo de estudio fue no experimental, de nivel descriptivo y correlacional y de diseño transversal, para el que se solicitó consentimiento informado a las directivas de los colegios seleccionados, a los padres de familia y a los estudiantes participantes.

\section{Procedimiento}

Los estudiantes diligenciaron en el aula de clase un formulario autoadministrado que incluía pun-

\section{TABLA 1}

Variables sociodemográficas de muestra

\begin{tabular}{|c|c|c|}
\hline Variable & Frecuencia & Porcentaje \\
\hline $\begin{array}{l}\text { Género } \\
\text { Hombre } \\
\text { Mujer }\end{array}$ & $\begin{array}{r}523 \\
432 \\
\end{array}$ & $\begin{array}{l}54.8 \\
45.2\end{array}$ \\
\hline $\begin{array}{l}\text { Edad } \\
14 \\
15 \\
16 \\
17 \\
18\end{array}$ & $\begin{array}{c}210 \\
272 \\
327 \\
135 \\
7 \\
\text { Sin dato (4) }\end{array}$ & $\begin{array}{c}22 \\
28.5 \\
34.2 \\
14.1 \\
0.7 \\
\text { Sin dato }(0.4)\end{array}$ \\
\hline $\begin{array}{l}\text { Estrato socioeconómico } \\
1-2-3 \\
4-5-6\end{array}$ & $\begin{array}{c}663 \\
273 \\
\text { Sin dato (19) }\end{array}$ & $\begin{array}{c}69.42 \\
28.58 \\
2 \\
\end{array}$ \\
\hline $\begin{array}{l}\text { Tipo de institución educativa } \\
\text { Pública } \\
\text { Privada }\end{array}$ & $\begin{array}{c}658 \\
287 \\
\text { Sin dato (10) }\end{array}$ & $\begin{array}{c}68.9 \\
30.1 \\
1\end{array}$ \\
\hline $\begin{array}{l}\text { Grado que cursan } \\
80 \\
9 \underline{0} \\
10^{\circ} \\
11^{\circ}\end{array}$ & $\begin{array}{c}59 \\
318 \\
280 \\
290 \\
\text { Sin dato (8) }\end{array}$ & $\begin{array}{c}6.2 \\
33.3 \\
29.3 \\
30.4 \\
0.8\end{array}$ \\
\hline
\end{tabular}

Fuente elaboración propia. 
tos que abordaban aspectos sociodemográficos, tipo de colegio, antecedentes familiares, actitudes cognitivas y afectivas en relación con el consumo de alcohol y drogas y prácticas sexuales, y variables relacionadas con los grupos.

Para el análisis descriptivo se utilizaron las distribuciones de frecuencia para las variables cualitativas y estadísticos descriptivos para las variables cuantitativas, previamente a la identificación de su distribución. La prueba de distribución usada fue la de Kolmogorov-Smirnov y se utilizaron medidas de posición y de resumen como la media y la desviación estándar, cuando la distribución era normal, mientras que para las distribuciones no normales se usaron la mediana y los valores mínimos y máximos para determinar el rango de variación de los datos.

En el análisis bivariado entre variables cualitativas, se utilizó la prueba chi cuadrado de independencia, y cuando las frecuencias esperadas fueron menos de cinco, la prueba exacta de Fisher. La decisión de rechazar la hipótesis nula de independencia entre las variables se tomó con base en el valor de $p$ menor o igual a 0.05. Cuando en análisis incluyó una variable cuantitativa y una cualitativa, se analizó con la prueba $U$ de Mann-Whitney, debido a que las variables cuantitativas no siguieron una distribución normal. Resultaron dos modelos explicativos de regresión logística teniendo como variables dependientes: 1) tener relaciones sexuales y 2) tener relaciones sexuales bajo el efecto de alcohol o drogas. Para elegir las variables que se incluirían en los modelos, se utilizó el criterio de Hosmer-Lemeshow. Para seleccionar el modelo explicativo, se llevó a cabo un procedimiento de selección por pasos, vía método de razón de verosimilitud, con el cual se obtuvieron los valores de los OR con sus respectivos intervalos de confianza de $95 \%$. La información se procesó con SPSS versión 19 y se editaron los resultados en Microsoft Excel.

\section{Resultados}

Los resultados de la investigación muestran una asociación estadísticamente significativa entre el sexo y el consumo de alcohol o drogas de los adolescentes entre los 14 y los 17 años, de colegios públicos y privados de la ciudad de Medellín $(p \leq 0.05)$. Los hombres consumen más alcohol o drogas que las mujeres, con valores de $82.2 \%$ para el género masculino y $73.6 \%$ para el femenino. El estrato socioeconómico en relación con el consumo de drogas también tiene significancia estadística

TABLA 2

Consumo de alcohol y drogas según el sexo, el estrato y el tipo de institución educativa

\begin{tabular}{lcccccc}
\hline \multirow{2}{*}{ Variables sociodemográficas } & \multicolumn{5}{c}{ iHas consumido alcohol o drogas? } & \multirow{2}{*}{ Valor p } \\
\cline { 3 - 6 } & & Sro. & $\%$ & Nro. & $\%$ & \\
\hline 1. Género & Masculino & 430 & 82.2 & 93 & 17.8 & 0.001 \\
& Femenino & 318 & 73.6 & 114 & 26.4 & \\
\hline 3. Estrato socioeconómico de & 1 & 80 & 72.1 & 31 & 27.9 & 0.007 \\
residencia & 2 & 222 & 73 & 82 & 27 & \\
& 3 & 204 & 82.3 & 44 & 17.7 & \\
& 4 & 138 & 82.1 & 30 & 17.9 & \\
& 5 & 72 & 84.7 & 13 & 15.3 & \\
& 6 & 19 & 95 & 1 & 5 & \\
& No respuesta & 13 & 68.4 & 6 & 31.6 & \\
\hline 4.Tipo de institución educativa & Pública & 494 & 75.1 & 164 & 24.9 & $<0.001$ \\
& Privada & 246 & 85.7 & 41 & 14.3 & \\
\hline
\end{tabular}

Nota. $p>0.05$.

Fuente elaboración propia. 
$(p \leq 0.05)$. Los estratos altos aparecen como los de mayor consumo: el estrato 6 con un $96 \%$, el estrato 5 con $84.7 \%$, los estratos 3 y 4 tienen consumos muy similares $83.3 \%$ y $82.1 \%$, respectivamente. Los estratos 1 y 2 , aunque con porcentajes altos $72.1 \%$ y 73 \% para cada caso, son los que menos consumen en comparación con los estratos altos. Los adolescentes de los colegios privados consumen más alcohol o drogas que los públicos: $85.7 \%$ en los primeros y $75.1 \%$ en los segundo $(p<0.01)$ (Tabla 2).

Los adolescentes de este estudio han sido influenciados alguna vez $(92.7 \%)$ o muchas veces (91.9\%), para consumir alcohol. Sobre su decisión de consumir drogas $(p<0.001)$, los adolescentes entre los 14 y los 17 años han sido presionados para hacerlo alguna vez (92.6\%) y muchas veces (90.7\%). También han sido presionados para tener prácticas sexuales bajo el efecto de alcohol o drogas, alguna vez (96.8 \%) y muchas veces $(87.8 \%$ ), mostrando esta respuesta una asociación estadística significativa de $p<0.01$ (Tabla 3).

Con respecto a las actitudes cognitivas y afectivas, se halló una relación significativa entre estas y el consumo de alcohol y drogas en los adolescentes. Las actitudes cognitivas relacionadas con creencias sobre las bondades y ventajas que tienen el alcohol y las drogas para mejorar el desempeño sexual, aumento de sensaciones, placer, autoestima o facilitar el inicio de las relaciones sexuales, tendrán un efecto directo sobre el hecho de consumir alcohol o drogas. En cuanto a las actitudes cognitivas, se observan también relaciones estadísticamente significativas con el consumo de alcohol y drogas; especialmente aquellas actitudes afectivas de contenidos asociados con el agrado, desagrado o aceptación de realizar prácticas sexuales o experimentarlas bajo el efectos del alcohol o las drogas (Tabla 4).

Los análisis multivariados mostraron dos modelos explicativos. En el modelo 1, se tomaron

TABLA 3

Variables relacionadas con los grupos de pares y consumo de alcohol o drogas

\begin{tabular}{|c|c|c|c|c|c|c|}
\hline \multirow{2}{*}{\multicolumn{2}{|c|}{ Variables relacionadas con los grupos: }} & \multicolumn{4}{|c|}{ ¿Has consumido alcohol o drogas? } & \multirow{3}{*}{ Valor $\mathrm{p}$} \\
\hline & & \multicolumn{2}{|c|}{ Sí } & \multicolumn{2}{|c|}{ No } & \\
\hline & & Nro. & $\%$ & Nro. & $\%$ & \\
\hline \multirow{3}{*}{$\begin{array}{l}\text { 1. Han influido en mi decisión para consu- } \\
\text { mir alcohol. }\end{array}$} & Nunca & 287 & 63.5 & 165 & 36.5 & $<0.001$ \\
\hline & Alguna vez & 358 & 92.7 & 28 & 7.3 & \\
\hline & Muchas veces & 102 & 91.9 & 9 & 8.1 & \\
\hline \multirow{3}{*}{$\begin{array}{l}\text { 2. Han influido en mi decisión para consu- } \\
\text { mir drogas. }\end{array}$} & Nunca & 514 & 74 & 181 & 26 & $<0.001$ \\
\hline & Alguna vez & 162 & 92.6 & 13 & 7.4 & \\
\hline & Muchas veces & 68 & 90.7 & 7 & 9.3 & \\
\hline \multirow{3}{*}{$\begin{array}{l}\text { 3. Han influido en mi decisión de tener } \\
\text { prácticas sexuales bajo el efecto de alcohol- } \\
\text { droga. }\end{array}$} & Nunca & 612 & 76.2 & 191 & 23.8 & $<0.001$ \\
\hline & Alguna vez & 90 & 96.8 & 3 & 3.2 & \\
\hline & Muchas veces & 36 & 87.8 & 5 & 12.2 & \\
\hline \multirow{3}{*}{$\begin{array}{l}\text { 4. Me han obligado a realizar algún tipo de } \\
\text { prácticas sexuales, bajo los efectos del alco- } \\
\text { hol-droga. }\end{array}$} & Nunca & 718 & 78.2 & 200 & 21.8 & 0.085 \\
\hline & Alguna vez & 18 & 94.7 & 1 & 5.3 & \\
\hline & Muchas veces & 7 & 100 & 0 & 0 & \\
\hline \multirow{3}{*}{$\begin{array}{l}\text { 5. Alguna persona estando bajo efectos del } \\
\text { alcohol-droga, me ha obligado a tener una } \\
\text { práctica sexual. }\end{array}$} & Nunca & 715 & 78.4 & 197 & 21.6 & 0.269 \\
\hline & Alguna vez & 23 & 85.2 & 4 & 14.8 & \\
\hline & Muchas veces & 7 & 100 & 0 & 0 & \\
\hline
\end{tabular}

$p>0.05$.

Fuente elaboración propia. 
TABLA 4

Creencias cognitivas y afectivas y consumo de alcohol y drogas en adolescentes

\begin{tabular}{|c|c|c|c|c|c|c|}
\hline \multirow{3}{*}{\multicolumn{2}{|c|}{$\begin{array}{l}\text { Variables psicosociales relacionadas con percepción de prácticas } \\
\text { sexuales y consumo de sustancias psicoactivas }\end{array}$}} & \multicolumn{4}{|c|}{ ¿Has consumido alcohol o drogas? } & \multirow{3}{*}{ Valor $\mathrm{p}$} \\
\hline & & \multicolumn{2}{|c|}{ Sí } & \multicolumn{2}{|c|}{ No } & \\
\hline & & Nro. & $\%$ & Nro. & $\%$ & \\
\hline \multirow{5}{*}{$\begin{array}{l}\text { Creo que las drogas y el alcohol mejo- } \\
\text { ran el desempeño sexual. }\end{array}$} & Totalmente de acuerdo & 24 & 85.7 & 4 & 14.3 & $<0.001$ \\
\hline & De acuerdo & 74 & 89.2 & 9 & 10.8 & \\
\hline & Indiferente & 188 & 83.2 & 38 & 16.8 & \\
\hline & En desacuerdo & 224 & 82.1 & 49 & 17.9 & \\
\hline & Totalmente en desacuerdo & 238 & 69 & 107 & 31 & \\
\hline \multirow{5}{*}{$\begin{array}{l}\text { Considero que las drogas y el alcohol } \\
\text { mejoran las sensaciones y la sensibili- } \\
\text { dad sexual. }\end{array}$} & Totalmente de acuerdo & 33 & 97.1 & 1 & 2.9 & $<0.001$ \\
\hline & De acuerdo & 94 & 90.4 & 10 & 9.6 & \\
\hline & Indiferente & 200 & 83. & 41 & 17 & \\
\hline & En desacuerdo & 222 & 81 & 52 & 19 & \\
\hline & Totalmente en desacuerdo & 198 & 66 & 102 & 34 & \\
\hline \multirow{5}{*}{$\begin{array}{l}\text { Pienso que tener prácticas sexuales } \\
\text { bajo el efecto de una droga o el alco- } \\
\text { hol es más placentero. }\end{array}$} & Totalmente de acuerdo & 46 & 93.9 & 3 & 6.1 & $<0.001$ \\
\hline & De acuerdo & 94 & 87 & 14 & 13 & \\
\hline & Indiferente & 219 & 83.3 & 44 & 16.7 & \\
\hline & En desacuerdo & 207 & 81.8 & 46 & 18.2 & \\
\hline & Totalmente en desacuerdo & 169 & 63.5 & 97 & 36.5 & \\
\hline \multirow{5}{*}{$\begin{array}{l}\text { El consumo de alcohol-drogas me } \\
\text { facilita tener prácticas sexuales. }\end{array}$} & Totalmente de acuerdo & 40 & 83.3 & 8 & 16.7 & 0.001 \\
\hline & De acuerdo & 124 & 86.7 & 19 & 13.3 & \\
\hline & Indiferente & 213 & 79.8 & 54 & 20.2 & \\
\hline & En desacuerdo & 205 & 80.1 & 51 & 19.9 & \\
\hline & Totalmente en desacuerdo & 161 & 68.8 & 73 & 31.2 & \\
\hline \multirow{5}{*}{$\begin{array}{l}\text { El consumo de drogas o alcohol me da } \\
\text { seguridad antes de la práctica sexual. }\end{array}$} & Totalmente de acuerdo & 19 & 95 & 1 & 5 & 0.001 \\
\hline & De acuerdo & 62 & 84.9 & 11 & 15.1 & \\
\hline & Indiferente & 188 & 79 & 50 & 21 & \\
\hline & En desacuerdo & 243 & 82.9 & 50 & 17.1 & \\
\hline & Totalmente en desacuerdo & 230 & 71.4 & 92 & 28.6 & \\
\hline \multirow{5}{*}{$\begin{array}{l}\text { Me siento más aceptado sexualmente } \\
\text { cuando consumo alcohol-drogas. }\end{array}$} & Totalmente de acuerdo & 17 & 89.5 & 2 & 10.5 & $<0.001$ \\
\hline & De acuerdo & 30 & 93.8 & 2 & 6.3 & \\
\hline & Indiferente & 184 & 83.6 & 36 & 16.4 & \\
\hline & En desacuerdo & 217 & 81.9 & 48 & 18.1 & \\
\hline & Totalmente en desacuerdo & 289 & 71.5 & 115 & 28.5 & \\
\hline \multirow{5}{*}{$\begin{array}{l}\text { Si mis amigos tienen prácticas sexua- } \\
\text { les bajo efectos de alcohol-drogas es } \\
\text { adecuado. }\end{array}$} & Totalmente de acuerdo & 10 & 90.9 & 1 & 9.1 & $<0.001$ \\
\hline & De acuerdo & 14 & 82.4 & 3 & 17.6 & \\
\hline & Indiferente & 232 & 87.2 & 34 & 12.8 & \\
\hline & En desacuerdo & 227 & 78.3 & 63 & 21.7 & \\
\hline & Totalmente en desacuerdo & 262 & 71.8 & 103 & 28.2 & \\
\hline
\end{tabular}




\begin{tabular}{|c|c|c|c|c|c|c|}
\hline \multirow{3}{*}{\multicolumn{2}{|c|}{$\begin{array}{l}\text { Variables psicosociales relacionadas con percepción de prácticas } \\
\text { sexuales y consumo de sustancias psicoactivas }\end{array}$}} & \multicolumn{4}{|c|}{ ¿Has consumido alcohol o drogas? } & \multirow{3}{*}{ Valor $\mathrm{p}$} \\
\hline & & \multicolumn{2}{|c|}{ Sí } & \multicolumn{2}{|c|}{ No } & \\
\hline & & Nro. & $\%$ & Nro. & $\%$ & \\
\hline \multirow{5}{*}{$\begin{array}{l}\text { Tener prácticas sexuales bajo los } \\
\text { efectos del alcohol y las drogas es una } \\
\text { experiencia gratificante. }\end{array}$} & Totalmente de acuerdo & 16 & 76.2 & 5 & 23.8 & $<0.001$ \\
\hline & De acuerdo & 84 & 94.4 & 5 & 5.6 & \\
\hline & Indiferente & 257 & 82.4 & 55 & 17.6 & \\
\hline & En desacuerdo & 205 & 80.7 & 49 & 19.3 & \\
\hline & Totalmente en desacuerdo & 182 & 67.4 & 88 & 32.6 & \\
\hline \multirow{5}{*}{$\begin{array}{l}\text { Consumir droga y alcohol me hace } \\
\text { más interesante y deseado sexual- } \\
\text { mente. }\end{array}$} & Totalmente de acuerdo & 12 & 80 & 3 & 20 & $<0.001$ \\
\hline & De acuerdo & 40 & 88.9 & 5 & 11.1 & \\
\hline & Indiferente & 169 & 79.7 & 43 & 20.3 & \\
\hline & En desacuerdo & 246 & 85.4 & 42 & 14.6 & \\
\hline & Totalmente en desacuerdo & 277 & 71.4 & 111 & 28.6 & \\
\hline \multirow{5}{*}{$\begin{array}{l}\text { Considero que cuando estoy drogado } \\
\text { (a) o bajo los efectos del alcohol soy } \\
\text { menos exigente al escoger mi pareja } \\
\text { sexual. }\end{array}$} & Totalmente de acuerdo & 94 & 89.5 & 11 & 10.5 & $<0.001$ \\
\hline & De acuerdo & 190 & 89.6 & 22 & 10.4 & \\
\hline & Indiferente & 184 & 73.9 & 65 & 26.1 & \\
\hline & En desacuerdo & 127 & 78.4 & 35 & 21.6 & \\
\hline & Totalmente en desacuerdo & 144 & 67.0 & 71 & 33.0 & \\
\hline \multirow{5}{*}{$\begin{array}{l}\text { Siento agrado cuando me estimulan } \\
\text { sexualmente cuando estoy bajo los } \\
\text { efectos del alcohol o la droga. }\end{array}$} & Totalmente de acuerdo & 30 & 90.9 & 3 & 9.1 & $<0.001$ \\
\hline & De acuerdo & 94 & 94 & 6 & 6 & \\
\hline & Indiferente & 191 & 80.6 & 46 & 19.4 & \\
\hline & En desacuerdo & 184 & 78.3 & 51 & 21.7 & \\
\hline & Totalmente en desacuerdo & 244 & 71.1 & 99 & 28.9 & \\
\hline \multirow{5}{*}{$\begin{array}{l}\text { Desearía tener alguna vez sexo bajo } \\
\text { los efectos de una droga o alcohol. }\end{array}$} & Totalmente de acuerdo & 54 & 94.7 & 3 & 5.3 & $<0.001$ \\
\hline & De acuerdo & 116 & 92.8 & 9 & 7.2 & \\
\hline & Indiferente & 184 & 88.9 & 23 & 11.1 & \\
\hline & En desacuerdo & 120 & 75.9 & 38 & 24.1 & \\
\hline & Totalmente en desacuerdo & 263 & 67.1 & 129 & 32.9 & \\
\hline \multirow{5}{*}{$\begin{array}{l}\text { Siento que disfruto más las prácticas } \\
\text { sexuales cuando estoy bajo el efecto } \\
\text { de las drogas o el alcohol. }\end{array}$} & Totalmente de acuerdo & 24 & 82.8 & 5 & 17.2 & $<0.001$ \\
\hline & De acuerdo & 49 & 96.1 & 2 & 3.9 & \\
\hline & Indiferente & 204 & 83.6 & 40 & 16.4 & \\
\hline & En desacuerdo & 183 & 80.6 & 44 & 19.4 & \\
\hline & Totalmente en desacuerdo & 281 & 71.3 & 113 & 28.7 & \\
\hline
\end{tabular}

$p>0.05$.

Fuente elaboración propia.

como variable dependiente las relaciones sexuales; dicho modelo incluyó un total de 893 casos (93.5\%) del total de la muestra $(N=955)$ y explicó el $29.3 \%$ de la varianza. En cuanto al género, los hombres tienen una probabilidad de riesgo de tener relaciones sexuales 2.5 veces mayor que las mujeres. El estrato 2 es el que muestra un mayor de tener relaciones sexuales en comparación con los demás estratos socioeconómicos. El tipo de familia mostró que los adolescentes de familia monoparental tienen 2.08 veces más probabilidades de tener prácticas sexuales de riesgo, comparados con los de familias nucleares. Según el tipo de persona encargada de la crianza de los adolescentes, 
TABLA 5

Modelo 1. Relaciones sexuales y variables psicosociales

\begin{tabular}{|c|c|c|c|c|c|c|c|}
\hline Características & Coeficiente & Error estándar & Wald & Valor $p$ & OR & \multicolumn{2}{|c|}{ IC $95 \%$} \\
\hline Edad & 0.3 & 0.1 & 11.7 & $<0.001$ & 1.317 & 1.124 & 1.543 \\
\hline Género Masculino & 1 & 0.2 & 33.4 & $<0.001$ & 2.587 & 1.874 & 3.57 \\
\hline \multicolumn{8}{|l|}{ Estrato socioeconómico } \\
\hline Estrato 1 & & & 26.8 & $<0.001$ & & \multicolumn{2}{|c|}{1} \\
\hline Estrato 2 & 0.1 & 0.3 & 0 & 0.83 & 1.06 & 0.622 & 1.805 \\
\hline Estrato 3 & -0.3 & 0.3 & 1.5 & 0.22 & 0.709 & 0.412 & 1.222 \\
\hline Estrato 4 & -1 & 0.3 & 11.7 & $<0.001$ & 0.357 & 0.198 & 0.644 \\
\hline Estratos 5 y 6 & -0.9 & 0.3 & 7.4 & 0.01 & 0.397 & 0.204 & 0.772 \\
\hline \multicolumn{8}{|l|}{ Tipo de familia } \\
\hline Nuclear & & & 10.2 & 0.04 & & \multicolumn{2}{|c|}{1} \\
\hline Extensa & 0.1 & 0.2 & 0.2 & 0.69 & 1.088 & 0.723 & 1.637 \\
\hline Monoparental femenina & 0.7 & 0.2 & 8.9 & $<0.001$ & 2.081 & 1.287 & 3.366 \\
\hline Monoparental masculina & 0.6 & 0.5 & 1.3 & 0.25 & 1.821 & 0.652 & 5.089 \\
\hline Otro & 0.3 & 0.4 & 0.6 & 0.43 & 1.382 & 0.617 & 3.095 \\
\hline \multicolumn{8}{|l|}{ Personas encargadas de la crianza } \\
\hline Progenitores & & & 20.9 & $<0.001$ & & \multicolumn{2}{|c|}{1} \\
\hline Madre sola o con familiares & 0 & 0.2 & 0 & 0.97 & 1.006 & 0.697 & 1.453 \\
\hline Padre solo o con familiares & 1.7 & 0.6 & 8 & $<0.001$ & 5.656 & 1.702 & 18.792 \\
\hline Abuelos solos o con otros familiares & 1.7 & 0.5 & 11 & $<0.001$ & 5.345 & 1.983 & 14.407 \\
\hline Otros familiares & 0.4 & 0.6 & 0.3 & 0.56 & 1.462 & 0.413 & 5.172 \\
\hline Otras personas & 1.7 & 1.2 & 2.1 & 0.14 & 5.47 & 0.562 & 53.238 \\
\hline \multicolumn{8}{|l|}{ Cumplir con las normas familiares } \\
\hline Siempre & & & 10.3 & 0.01 & & \multicolumn{2}{|c|}{1} \\
\hline Algunas veces & 0.6 & 0.2 & 10.2 & $<0.001$ & 1.778 & 1.249 & 2.531 \\
\hline Nunca & 0.6 & 0.5 & 1.3 & 0.26 & 1.855 & 0.632 & 5.443 \\
\hline \multicolumn{8}{|c|}{ El no cumplir las normas en su familia ocasiona } \\
\hline Diálogo & & & 8.2 & 0.02 & & \multicolumn{2}{|c|}{1} \\
\hline Castigo & -0.4 & 0.2 & 4.7 & 0.03 & 0.691 & 0.495 & 0.966 \\
\hline Nada & 0.5 & 0.3 & 2.1 & 0.15 & 1.613 & 0.846 & 3.076 \\
\hline Actitudes cognitivas & 0 & 0 & 6.2 & 0.01 & 1.026 & 1.006 & 1.048 \\
\hline Consumo de alcohol o drogas & 1.4 & 0.2 & 48 & $<0.001$ & 4.016 & 2.711 & 5.951 \\
\hline Constante & -6.4 & 1.3 & 23 & $<0.001$ & 0.002 & & \\
\hline
\end{tabular}

Nota. OR = Odds Ratio; IC = Intervalo de confianza.

Fuente elaboración propia.

se encontró que cuando esta es responsabilidad del padre u otros familiares, se incrementa la probabilidad de tener prácticas sexuales inseguras en 5.6 veces en comparación con aquellos adolescentes criados por sus dos progenitores. Las actitudes cognitivas en los adolescentes que favorecen el consumo de alcohol y drogas aumentan también (1.02 veces) la probabilidad de tener prácticas sexuales inseguras (Tabla 5).
En el modelo 2 se tomaron las relaciones sexuales bajo el consumo de alcohol o drogas como variable dependiente. En este modelo se incluyeron 461 casos, y tuvo un porcentaje de explicación de la varianza del $13.9 \%$. Los resultados muestran tres variables independientes como factores explicativos y de riesgo en los adolescentes para tener relaciones sexuales bajo los efectos del alcohol y las drogas (la edad, la presión de grupo y las actitudes afectivas). 
Los datos relacionados con la edad señalan que la edad de inicio de consumo incrementa el riesgo de que el adolescente tenga prácticas sexuales inseguras bajo los efectos de las drogas y el alcohol en 1.35 veces. La presión de grupo se relaciona con prácticas sexuales bajo los efectos del alcohol. En comparación de adolescentes que nunca han sido presionados, los que lo han sido muchas veces tienen 2.9 veces más probabilidad de tener prácticas sexuales de riesgo. Finalmente, aquellas tendencias afectivas que impliquen algún grado de aceptación o de valoración positiva sobre prácticas sexuales bajo el efecto de alcohol o drogas, aumentará en 1.09 veces la probabilidad de que el adolescente use alcohol o drogas para tener prácticas sexuales (Tabla 6).

\section{Discusión y conclusiones}

Los resultados del presente estudio permiten establecer algunas inferencias con respecto a factores de riesgo durante la adolescencia, relacionados con el consumo de alcohol o drogas y prácticas sexuales.

1. La descripción de variables personales como la edad, el sexo y las actitudes cognitivas y afectivas, indican altos riesgos en la adolescencia que aumentan la probabilidad de consumo de alcohol y drogas y de tener prácticas sexuales inseguras. Las actitudes cognitivas y afectivas durante la infancia y la adolescencia son los cimientos psicológicos básicos para la aparición de conductas de alto riesgo relacionadas con el consumo de sustancias y la realización de prácticas sexuales inseguras durante la adolescencia y la adultez.

2. Variables como el tipo de familia, las personas encargadas de la crianza y las normas al interior del grupo familiar, juegan un papel fundamental en la aparición y desarrollo de conductas de alto riesgo para el consumo y conductas sexuales a temprana edad durante la adolescencia.

3. El papel que tienen variables relacionales como el grupo de pares (amigos y compañeros del colegio), serían un factor de riesgo significativo para el uso de alcohol y drogas en las prácticas sexuales de los adolescentes.

La edad como factor de riesgo personal para el consumo de alcohol o drogas y conductas sexuales inseguras, se describe en esta investigación como una variable que está presente a muy temprano inicio de la adolescencia, particularmente a los 13 años. Autores como Grant, Stinson y Harford (2001) plantean una relación entre el inicio del consumo de alcohol o drogas y problemáticas relacionadas con la iniciación temprana. Así, la edad de inicio de consumo en los adolescentes del presente estudio puede considerarse como un predictor del tipo de problemas que pueden acompañar el consumo, como por ejemplo el inicio temprano de prácticas sexuales de riesgo, el no uso de métodos de anticoncepción o protección, los contagios por infecciones de transmisión sexual o los embarazos no deseados y de alto riesgo. Con respecto al desarrollo de los adolescentes y relacionado con la edad,

TABLA 6

Modelo 2. Relaciones sexuales bajo el efecto de alcohol o drogas y variables psicosociales

\begin{tabular}{cccccccc}
\hline \multicolumn{1}{c}{ Características } & Coeficiente & Error estándar & Wald & Valor $\mathbf{p}$ & OR & \multicolumn{2}{c}{ IC 95\% } \\
\hline Edad & 0.3 & 0.1 & 8.7 & $<0.001$ & 1.353 & 1.107 & 1.654 \\
\hline Influencia de los grupo en la decisión de tener prácticas sexuales bajo alcohol o drogas \\
$\quad$ Nunca \\
Alguna vez & 0.9 & 0.3 & 13.9 & $<0.001$ & & & \\
$\quad$ Muchas veces & 1.1 & 0.5 & 5.6 & 0.02 & 2.912 & 1.204 & 7.041 \\
& 0.1 & 0 & 24.7 & $<0.001$ & 1.095 & 1.056 & 1.134 \\
Actitudes afectivas & -5.8 & 1.7 & 12.5 & $<0.001$ & 0.003 & & \\
Constante & & &
\end{tabular}

Nota. OR = Odds Ratio; IC = Intervalo de confianza.

Fuente elaboración propia. 
algunos autores proponen que a menor edad y bajo la acción de ciertas variables ambientales como la familia, los amigos, el barrio donde viven, entre otros, se aumenta la probabilidad en el adolescente de adquisición de conductas problemáticas (Campo et al., 2010).

La familia y las características que se encuentran alrededor de esta, como la crianza y las normas, son en este estudio un factor de riesgo importante para la aparición de consumo temprano de alcohol o drogas y el inicio de prácticas sexuales en los adolescentes. Según los resultados obtenidos, los adolescentes de familias de estructura monoparental tienen mayor probabilidad (2.08 veces) de tener prácticas sexuales bajo el efecto del alcohol y las drogas.

Con respecto a la familia, pero también con las variables cognitivas y afectivas del adolescente (sus actitudes), gran parte del contenido del sistema de creencias del adolescente está representado en las pautas de socialización y educación que recibe al interior del seno familiar. Las prácticas pedagógicas con las que los padres orientan a sus hijos adolescentes tienen un efecto socializante de normas y pautas de conducta que servirán como guía para la toma de decisiones de un sinnúmero de situaciones de alto riesgo al que se expone el adolescente (Baumrind, 1978).

Los resultados del presente trabajo aportan evidencias que apoyan dichas afirmaciones. El contenido de las actitudes cognitivas y afectivas está relacionado con ciertas características familiares de los adolescentes: así, adolescentes que provienen de familias monoparentales, con problemas de abuso de sustancias y existencia de promiscuidad de uno de sus padres, aborto, infecciones de transmisión sexual, tienen tendencia a percibir de una manera natural dichas características de su entorno familiar, generándose, posiblemente, mecanismos cognitivos y afectivos que alimentan y consolidan creencias y tendencias emocionales que faciliten al adolescente realizar prácticas sexuales bajo los efectos de sustancias psicoactivas.

Estas actitudes cognitivas y afectivas juegan un papel central en la toma de decisiones y las conductas en los adolescentes en cuanto a las prácticas sexuales bajo los efectos del alcohol o drogas. En el estudio actual se halló una relación estrecha entre las actitudes cognitivas y afectivas favorables para el consumo de alcohol y drogas y prácticas sexuales bajo sus efectos. Los adolescentes que expresan creencias sobre las ventajas y bondades de las drogas o el alcohol en las prácticas sexuales, son los que mayor riesgo tienen de que estas sean inseguras y conlleven a infecciones de transmisión sexual, embarazos no deseados, abortos y no utilización de métodos anticonceptivos y de protección. Algunos autores apoyan estos resultados (Bennett \& Ames, 1985; Cano, Berjano \& Jabakhanji, 1988; Eiser, Morgan, Gammaget \& Gray, 1989; Moral \& Ovejero, 2005) y sugieren que las actitudes permisivas son un predictor de la conducta de consumo durante edades tempranas en el desarrollo de un individuo.

Este estudio abre la posibilidad de generar nuevas líneas de investigación en el estudio de los componentes cognitivos, afectivos y psicosociales implicados en el consumo de sustancias psicoactivas y prácticas sexuales inseguras durante la adolescencia. Los resultados obtenidos apuntan hacia la descripción de un modelo de factores de riesgo psicosociales relacionados con las prácticas sexuales inseguras bajo el consumo de sustancias psicoactivas.

En síntesis, y según los resultados de esta investigación, se considera que ser un adolescente de género masculino de estrato 2 , proveniente de una familia de estructura monoparental, con actitudes cognitivas y afectivas relacionadas con percepciones favorables hacia los beneficios que las drogas tienen sobre el desempeño en las relaciones sexuales, el agrado y aceptación de usar sustancias psicoactivas durante las relaciones sexuales y las percepciones y la falta de información sobre métodos anticonceptivos y de protección, son en conclusión el conjunto de variables psicosociales más representativas que explican para esta muestra, el fenómeno de las prácticas sexuales bajo el consumo de sustancias psicoactivas de adolescentes de la ciudad de Medellín en el año 2011.

No obstante, cabe anotar, que estas variables no serían las únicas que pudieran generar dichas prácticas riesgosas; estudios futuros, podrán considerar otras variables independientes que no se hayan 
tenido en cuenta en esta investigación, tales como el barrio donde viven los adolescentes, los antecedentes psicológicos y psiquiátricos y la comorbilidad con trastornos de conducta y afectivos, entre otros.

\section{Referencias}

Abasiubong, F. U., Udoh, S. B., Ntuk, E. A., Ekanem, U. S. \& Etukumana, E. A. (2011). Gender differences in attitude to use of condom and sexual practices among higher school students in Nigeria. TAF Preventive Medicine Bulletin, 10(5), 519-532.

Ajzen, I. \& Fishbein, F. (2008). Scaling and testing multiplicative combinations in the expectancyvalue model of attitudes. Journal of Applied Social Psychology, 38(9), 2222-2247. doi: 10.1111/j.1559. 1816.2008.00389

Ajzen, I. \& Gilbert Cote, N. (2008). Attitudes and prediction of behavior. En W. D. Crano \& R. Prislin (Eds.), Attitudes and attitude change (pp. 239-311). New York: Psychology Press.

Baumrind, D. (1978). Parental disciplinary patterns and social competence in children. Youth and Society, 9(3), 239-267. doi: 10.1177/0044118X7800900302

Bennett, L. A. \& Ames, G. M. (1985). The American experience with alcohol: Contrasting cultural perspectives. Nueva York: Plenum.

Bertoni, N., Bastos, F. I., Mello, M. B., Makuch, M. Y., Sousa, M. H., Osis, M. J., et al. (2009). Alcohol and illicit drug use and its influence on the sexual behavior of teenage from Minas Gerais State, Brazil. Cuadernos de Saúde Pública, 25(6), 1350-1360. Recuperado el 13 de agosto de 2011, de http:// dx.doi.org/10.1590/S0102-311X2009000600017

Caldeira, K. M., Arria, A. M., O'Grady, K. E., Zarate, E. M., Vincent, K. B. \& Wixh, E. D. (2009). Prospective associations between alcohol and drug consumption and risky sex among female college students. Journal of Alcohol and Drug Education, 53(2), 171-192. Recuperado el 13 de agosto de 2011, de http://www.ncbi.nlm.nih.gov/pmc/articles/PMC2806636/pdf/nihms115858.pdf

Calsyn, D. A., Cousing, S. J., Hatch-Maillette, M. A., Forcehimes, A., Mandler, R., Doyle, S. R., et al. (2010). Sex under the influence of drugs or alcohol: Common for men in substance abuse treatment and associated with high-risk sexual behavior. American Journal on Addictions, 19(2), 119-127. doi: 10.1111/j.1521-0391.2009.00022.x

Campo, A. A. (2009). Relaciones sexuales en adolescentes colombianos y las implicaciones para la salud pública: una revisión de la prevalencia y algunas variables asociadas. MedUNAB, 12, 86-90. Recuperado el 19 de agosto de 2011, de http://www. sidastudi.org/resources/inmagic-img/DD9739.pdf

Campo, A. A., Ceballo, G. A. \& Henazo, E. (2010, marzo-abril). Prevalencia de patrón de comportamiento de riesgo para la salud sexual y reproductiva en estudiantes adolescentes. Revista LatinoAmericana de Enfermagen, 18(2). Recuperado el 25 de agosto de 2011, de http://www.scielo.br/pdf/ rlae/v18n2/es_05.pdf

Cano, P. L., Berjano, P. E. \& Jabakhanji, R. H. (1988). El uso de drogas entre la población escolar. Valencia: Direcció General de Serveis Socials, Generalitat Valenciana.

Ceballos, G. A. \& Campo Arias, A. (2005). Prevalencia de uso de condón en la primera relación sexual en adolescentes de Santa Marta, Colombia: diferencias por género. Revista MED UNAB, 8(2), 59-64. Recuperado el 25 de agosto de 2011, de http://www. sidastudi.org/resources/inmagic-img/dd4276.pdf

Echeverría, S. F. (2009). ¿Qué conocimientos, actitudes y prácticas sexuales tienen los y las estudiantes de la licenciatura en Educación Física de Yucatán, ante el VIH/SIDA? Archivos Hispanoamericanos de Sexología, 15(2), 13-36.

Eiser, J. R., Morgan, M., Gammaget, P. \& Gray, E. (1989). Adolescent smoking: Attitudes, norms and parental influence. British Journal of Social Psychology, 28(3), 193-202.

Grant, B. F., Stinson, F. S. \& Harford, T. C. (2001). Age at onset of alcohol use and DSM-IV alcohol abuse and dependence: A 12-year follow-up. Journal of Substance Abuse, 13(4), 493-504.

Goncalvez, M. F., Da Silveira, D. X. \& Bartex, A. S. (2009). Knowledge and attitudes related to drug abuse and prevention displayed by public school educators. Revista Brasilera de Psiquiatría, 31(2), 95-100. Recuperado el 3 de septiembre de 2011, de http://www.scielo.br/pdf/rbp/v31n2/v31n2a03.pdf 
Jessor, R. (1991). Risk behavior in adolescence: A psychological framework for understanding and action. Journal of Adolescent Health, 12(8), 597-605. Recuperado el 3 de septiembre de 2011, de http:// www.colorado.edu/ibs/jessor/pubs/ 1991_Jessor_ JAH_RiskBehaviorinAdolescence.pdf

Jessor, R. \& Jessor, S. L. (1977). Problem behavior and psychosocial development: A longitudinal study of youth. New York: Academic Press.

Kafaar, Z., Swartz, L., Kagee, A., Lesch, A. \& Jaspan, H. (2007). Adolescent participation in HIV vaccine trials: Cognitive developmental considerations. South African Journal of Psychology, 37(3), 576-594.

L'Engle, K. L. \& Jackson, C. (2008). Socialization influences on early adolescents' cognitive susceptibility and transition to sexual intercourse. Journal of Research on Adolescence, 18(2), 353-37. Recuperado el 3 de septiembre de 2011, http://teenmedia.unc. edu/pdf/Socialization\%20Influences.pdf

Lameiras, F., Failde, G., Bimbela, P. \& Alfaro, (2008). Uso del preservativo masculino en las relaciones con coito vaginal de jóvenes españoles entre catorce y veinticuatro años. Revista Diversitas: Perspectivas en Psicología, 4(2), 401-415. Disponible en http://www.scielo.unal.edu.co/ scielo.php?script $=$ sci_arttext\&pid $=S 1794$ 99982008000200014\&lng $=\mathrm{es} \& \mathrm{nrm}=$

Mann, L., Harmoni, R. \& Power, C. (1989). Adolescent decision-making: The development of competence. Journal of Adolescence, 12(3), 265-278.

Meneses, C., Romo, N., Uroz, J., Gil, E., Markez, I., Gimenez, S., et al. (2009). Adolescencia, consumo de drogas y comportamientos de riesgo: diferencias por sexo, etnicidad y áreas geográficas en España. Trastornos Adictivos, 11(1), 51-63. Recuperado el 3 de septiembre de 2011, de http://www.ugr. es/ esmujer/Paginas\%20Personales/ EugeniaGil/pdf/TrastornosAdictivos2009.pdf

Moral, M. V. \& Ovejero, A. (2005). Modificación de las actitudes, los hábitos y frecuencia de consumo de alcohol y otras sustancias psicoactivas en adolescentes españoles a partir de un programa educativo-preventivo. Revista Colombiana de Psicología, 14(1), 100-118. Recuperado el 12 de septiembre de 2011, de http://redalyc.uaemex.mx/src/inicio/ ArtPdfRed.jsp?iCve $=80401410$

Orchowski, L. M. \& Barnett, N. P. (2012). Alcoholrelated sexual consequences during the transition from high school to college. Addictive Behaviors, 37(3), 256-263.

Patrick, M. E. \& Maggs, J. L. (2009). Does drinking lead to sex? Daily alcohol-sex behaviors and expectancies among college students. Psychology of Addictive Behaviors, 23(3), 472-481.

Sierra, D. R., Pérez, M., Pérez, A. \& Núñez, M. (2005). Representaciones sociales en jóvenes consumidores y no consumidores de sustancias psicoactivas. Adicciones, 17(4), 349-360. Recuperado el 13 de septiembre de 2011, de http://www.adicciones. es/files/Perez.pdf

Varela, M. T., Salazar, I. C., Cáceres, D. E. \& Tovar, J. R. (2007). Consumo de sustancias psicoactivas en jóvenes: factores psicosociales asociados. Pensamiento Psicológico, 3(8), 31-45. 